\title{
Erratum to: Profile of European adults interested in internet-based personalised nutrition: the Food4Me study
}

\author{
Katherine M. Livingstone ${ }^{1}$ - Carlos Celis-Morales ${ }^{1} \cdot$ Santiago Navas-Carretero $^{2,3}$. \\ Rodrigo San-Cristobal ${ }^{2,3}$. Clare B. O'Donovan ${ }^{4} \cdot$ Hannah Forster ${ }^{4}$. \\ Clara Woolhead $^{4} \cdot$ Cyril F. M. Marsaux ${ }^{5}$ Anna L. Macready ${ }^{6} \cdot$ Rosalind Fallaize $^{6}$. \\ Silvia Kolossa ${ }^{7} \cdot$ Lydia Tsirigoti $^{8} \cdot$ Christina P. Lambrinou $^{8} \cdot$ George Moschonis $^{8}$. \\ Magdalena Godlewska ${ }^{9} \cdot$ Agnieszka Surwiłło $^{9}$ Christian A. Drevon ${ }^{10}$ • \\ Yannis Manios ${ }^{8} \cdot$ Iwona Traczyk $^{9} \cdot$ Eileen R. Gibney ${ }^{4}$ Lorraine Brennan ${ }^{4}$. \\ Marianne C. Walsh $^{4} \cdot$ Julie A. Lovegrove $^{6} \cdot$ J. Alfredo Martinez $^{2,3} \cdot$ Wim H. Saris $^{5}$. \\ Hannelore Daniel $^{7} \cdot$ Mike Gibney $^{4} \cdot$ John C. Mathers ${ }^{1}$
}

Published online: 12 March 2016

(c) Springer-Verlag Berlin Heidelberg 2016

\section{Erratum to: Eur J Nutr DOI 10.1007/s00394-015-0897-y}

In the original published article Fig. 1 is incorrect.

The correct version of the Fig. 1 is given below:

The online version of the original article can be found under doi:10.1007/s00394-015-0897-y.

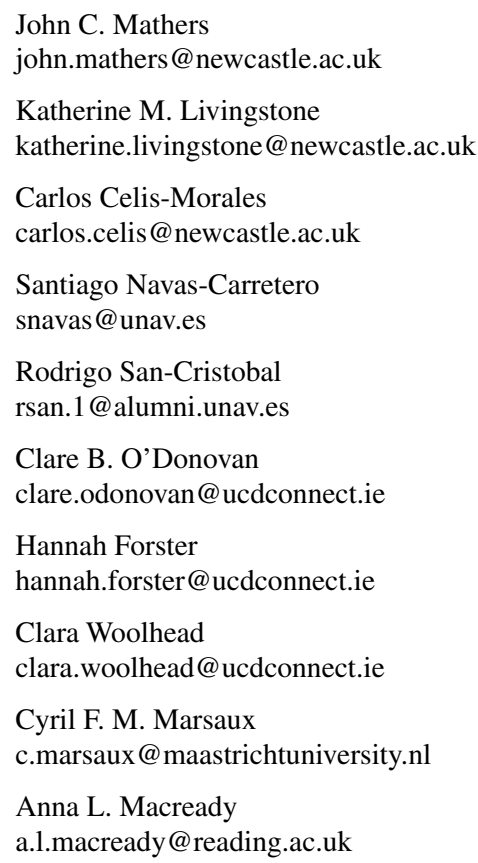

Rosalind Fallaize

r.fallaize@ reading.ac.uk

Silvia Kolossa

silvia.kolossa@tum.de

Lydia Tsirigoti

tsirigoti.lydia@gmail.com

Christina P. Lambrinou cplambrinos@gmail.com

George Moschonis gmoschi@hua.gr

Magdalena Godlewska mgodlewska@izz.waw.pl

Agnieszka Surwiłło asurwillo@izz.waw.pl

Christian A. Drevon c.a.drevon@medisin.uio.no

Yannis Manios manios@hua.gr

Iwona Traczyk itraczyk@izz.waw.pl 


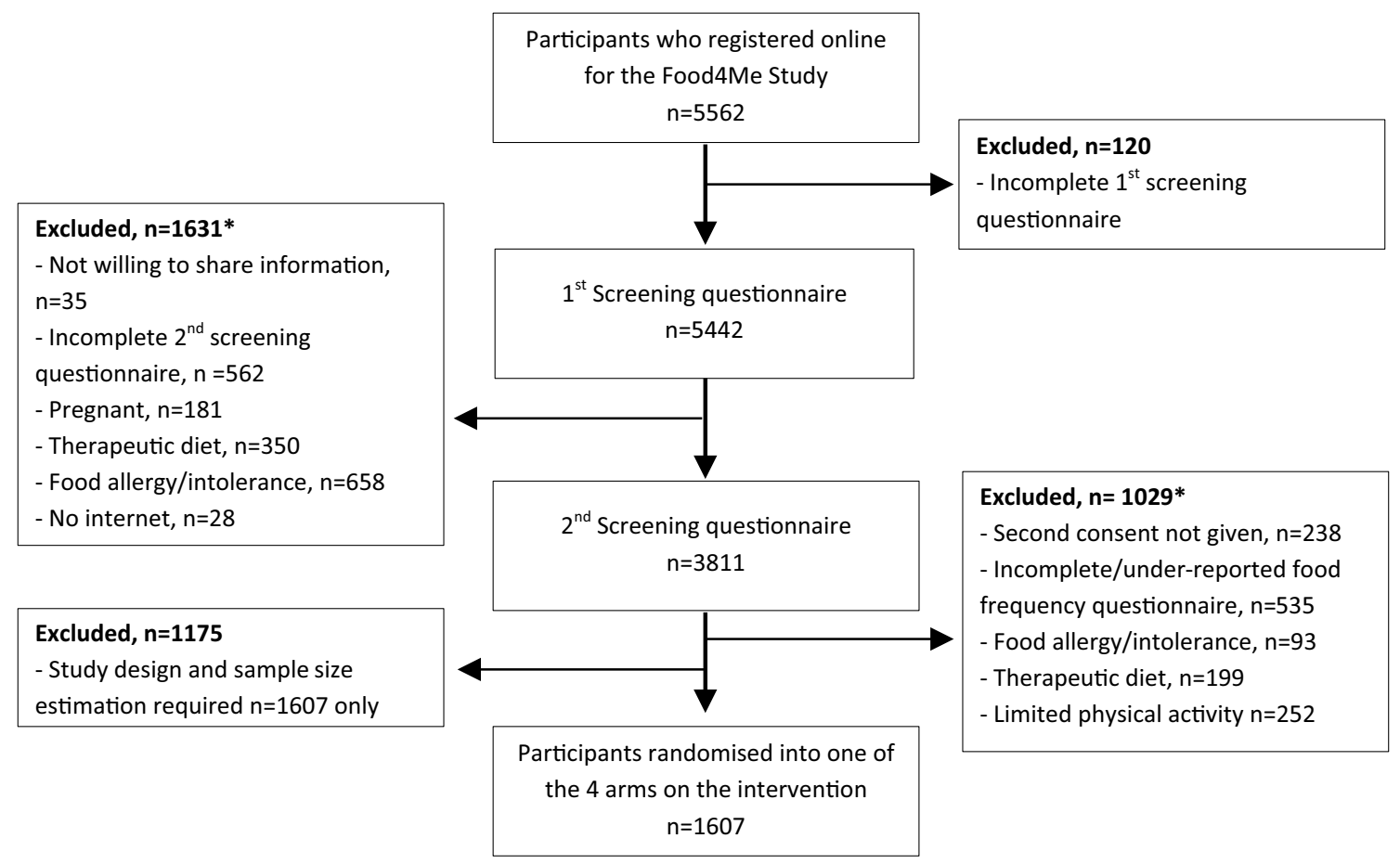

Fig. 1 Food4Me proof of principle study flow chart. Asterisk total number of participants reporting one or more exclusion criteria

Eileen R. Gibney

eileen.gibney@ucd.ie

Lorraine Brennan

lorraine.brennan@ucd.ie

Marianne C. Walsh

marianne.walsh@ucd.ie

Julie A. Lovegrove

j.a.lovegrove@reading.ac.uk

J. Alfredo Martinez

jalfmtz@unav.es

Wim H. Saris

w.saris@maastrichtuniversity.nl

Hannelore Daniel

hannelore.daniel@tum.de

Mike Gibney

mike.gibney@ucd.ie

1 Human Nutrition Research Centre, Institute of Cellular Medicine, Newcastle University, Biomedical Research Building, Campus for Ageing and Vitality, Newcastle upon Tyne NE4 5PL, UK
2 Center for Nutrition Research, University of Navarra, Pamplona, Spain

3 CIBER Fisiopatología Obesidad y Nutrición (CIBERobn), Instituto de Salud Carlos III, Madrid, Spain

4 UCD Institute of Food and Health, University College Dublin, Belfield, Dublin 4, Republic of Ireland

5 Department of Human Biology, NUTRIM School for Nutrition, Toxicology and Metabolism, Maastricht University Medical Centre, Maastricht, The Netherlands

6 Hugh Sinclair Unit of Human Nutrition and Institute for Cardiovascular and Metabolic Research, University of Reading, Reading, UK

7 ZIEL Research Center of Nutrition and Food Sciences, Biochemistry Unit, Technische Universität München, Munich, Germany

8 Department of Nutrition and Dietetics, Harokopio University, Athens, Greece

9 National Food and Nutrition Institute, (IZZ), Warsaw, Poland

10 Department of Nutrition, Institute of Basic Medical Sciences, Faculty of Medicine, University of Oslo, Oslo, Norway 